Admission and 30-day mortality: Both PHT and NVL were independently predictive, with PHT having the stronger effect and with no significant interaction (table 1).

For transfusion, PHT was strongly predictive but there was a highly significant interaction $(\mathrm{P}<0.001)$ such that NVL was associated with increased odds of transfusion in patients without PHT and reduced odds in those with PHT (table 1). The percentages requiring transfusion were: no PHT/NVL 20\%, NVL alone 33\%, PHT alone 57\%, PHT+NVL 44\%.

Conclusions There is a mismatch between the transfusion needs and clinical outcome in patients with both PHT and NVL, which may indicate that the bleeding is in some cases attributable to the NVL alone while the clinical outcome is related to the combined risk factors.

\section{PTU-069 ANTIPLATELET VERSUS ANTICOAGULANT EFFECTS IN NON-VARICEAL UPPER GASTROINTESTINAL BLEEDING} ${ }^{1,2}{ }^{2}$ Ali Taha* ${ }^{*}{ }^{1}$ Caroline McCloskey, ${ }^{1}$ Theresa Craigen, ${ }^{1}$ Wilson Angerson. ${ }^{1}$ University
Hospital Crosshouse, Kilmarnock, Kilmarnock, UK; ${ }^{2}$ University of Glasgow Medical School,
Glasgow, UK

\subsection{6/gutjnl-2019-BSGAbstracts.285}

Introduction Non-variceal upper gastrointestinal bleeding (NVUGIB) typically originates from mucosal damage or ulceration. The situation can be complicated by the wide use of antiplatelet agents (APs) and anticoagulants (ACs). These drugs, e.g. aspirin, may directly cause ulcers or they may provoke bleeding or re-bleeding. We aimed to assess the individual impacts of AP vs. AC activities on the clinical outcomes of NVUGIB.

Methods Patients with NVUGIB were sub-grouped according to their use of APs, ACs, or none (controls). They were excluded if they had portal hypertension or used NSAIDs. APs included aspirin, clopidogrel, ticagrelor, and dipyridamole. ACs included warfarin, heparin, and apixaban. A composite endoscopy score was devised. Clinical details were recorded including Blatchford score, admission, blood transfusion, rebleeding, and 30-day mortality. The Kruskal-Wallis or chi squared tests were used as appropriate. Odds ratios (ORs), comparing outcomes in AP and AC patients with controls, were calculated and adjusted for age, sex and endoscopy score using logistic regression.

Results The details of patients with NVUGIB (N=3233) including users of APs, ACs, and controls, who presented 2005-2016, are shown in table 1. The AP and AC groups were older and had worse clinical outcomes than controls.

When ORs were adjusted for age, sex and endoscopy score, APs and ACs were still more likely to be admitted [APs: OR (95\% CI), 1.38 (1.10-1.74), P=0.006; ACs: 3.48 (2.01-6.02), $\mathrm{P}<0.001$ ], and transfused [APs: 1.38 (1.15-1.66), $\mathrm{P}=0.001$; ACs: 2.53 (1.87-3.43), $\mathrm{P}<0.001]$. However, rebleeding remained significant only in ACs [2.26 (1.58-3.25), $\mathrm{P}<0.001]$, and neither APs nor ACs had increased mortality [APs: 0.75 (0.50-1.12), $\quad \mathrm{P}=0.16$; ACs: 1.00 (0.53-1.88), $\mathrm{P}=1.00]$.

Conclusions In NVUGIB, the antiplatelet and anticoagulant activities are associated with older age and greater need for admission, transfusion, and re-bleeding. These outcomes were more marked in users of anticoagulants even after adjustment for age, sex, and endoscopy, but with insignificant effect on mortality.
Abstract PTU-069 Table 1 The clinical details and outcomes of NVUGIB in users of Antiplatelets (APs), Anticoagulants (ACs), and controls

\begin{tabular}{llllll}
\hline & $\begin{array}{l}\text { Control } \\
\mathrm{n}=1976\end{array}$ & $\begin{array}{l}\text { Antiplatelets } \\
\mathrm{n}=1039\end{array}$ & $\begin{array}{l}\text { Anticoagulants } \\
\mathrm{n}=218\end{array}$ & $\begin{array}{l}\mathrm{P} \text { value } \\
\text { Overall }\end{array}$ & $\begin{array}{l}\mathrm{P} \\
\text { value } \\
\text { AP v } \\
\text { AC }\end{array}$ \\
\hline $\begin{array}{lllll}\text { Age, years, median } \\
\text { (IQR) }\end{array}$ & $54(39-70)$ & $75(66-82)$ & $75(66-83)$ & $<0.001$ & 0.92 \\
Blatchford score & $4(1-8)$ & $7(4-11)$ & $8(4-11)$ & $<0.001$ & 0.073 \\
Endoscopy score & $1(0-2)$ & $1(0-2)$ & $1(0-2)$ & 0.002 & 0.11 \\
Admitted & 1463 & $889(85.6 \%)$ & $203(93.1 \%)$ & $<0.001$ & 0.002 \\
& $(74.1 \%)$ & & & & \\
Transfused & 436 & $408(39.6 \%)$ & $114(52.5 \%)$ & $<0.001$ & 0.001 \\
& $(22.1 \%)$ & & & & \\
Re-bleeding & $186(9.5 \%)$ & $158(15.3 \%)$ & $54(24.9 \%)$ & $<0.001$ & 0.001 \\
Died $\leq 30$ days & $61(3.1 \%)$ & $50(4.8 \%)$ & $13(6.0 \%)$ & 0.015 & 0.50 \\
\hline
\end{tabular}

\section{PTU-070 NON-SPECIFIC UPPER GI MURAL THICKENING ON CT - IS IT JUST FROM PERISTALSIS?}

Neel Jain, Raunak Poonawala, Ruhaid Khurram, Rajan Patel ${ }^{*}$, Zohaib Tariq, Jonathan King, Kalpesh Besherdas. Royal Free London NHS Trust, UK

\subsection{6/gutjnl-2019-BSGAbstracts.286}

Introduction BSG published guidance on the indications for diagnostic endoscopy in April 2013, including abnormal or suspicious findings on CT imaging. Non-specific upper GI mural thickening on CT is a common abnormal finding raising the suspicion of upper GI malignancy. The correlation between CT mural thickening in the upper GI tract and endoscopic diagnosis of malignancy is not clearly known.

Methods A retrospective single centre study of patients referred for gastroscopy with the indication of 'abnormal imaging' $(n=147)$ was performed. Data was collected using the endoscopy software audit tool over a 3-year period (2016 to 2018). Patients with a CT reported finding of 'mural thickening' were included for analysis $(n=59)$. Statistics were performed using Welch's t-test.

Results 59 patients underwent gastroscopy for CT reported mural thickening: oesophageal 20 (34\%), GOJ 9 (15\%), gastric $23(39 \%)$, pyloric $4(7 \%)$, duodenal $5(8 \%)$ and jejunal 1 (2\%). Median time from CT to endoscopy was 21 days (IQR 12-54). Median age was 77 (IQR 62-83). Initial indication for CT scan included: weight loss 16 (27\%), abdominal pain 14 (24\%), possible malignancy $6(10 \%)$ and dysphagia 3 (5\%).

11 (19\%) patients had a normal gastroscopy, 24 (41\%) showed inflammatory changes (oesophagitis or gastritis), 20 (34\%) had evidence of a hiatus hernia, and 5 (8\%) had benign polyps.

$5(9 \%)$ had a histological diagnosis of gastric adenocarcinoma, $4(7 \%)$ of Barrett's oesophagus and 1 (2\%) of squamous dysplasia.

The 5 patients with adenocarcinoma could not be reliably identified by indication for imaging ( 2 for abdominal pain, 1 for weight loss, and 2 for non GI or systemic related symptoms).

The mean haemoglobin for the patients with malignancy was $104 \mathrm{~g} / \mathrm{L}$ vs $125 \mathrm{~g} / \mathrm{L}$ for the overall study group $(\mathrm{p}=0.13$, NS). The mean albumin for the patients with malignancy was 